\title{
Potential theory and approximation: highlights from the scientific work of Stephen Gardiner
}

\author{
Marius Ghergu ${ }^{1} \cdot$ Myrto Manolaki $^{1} \cdot$ Ivan Netuka $^{2} \cdot$ Hermann Render $^{1}$
}

Received: 25 February 2019 / Accepted: 25 April 2019 / Published online: 17 June 2019

(c) The Author(s) 2019

\section{Abstract}

This survey is dedicated to the scientific achievements of Stephen Gardiner in honour of his 60th birthday.

Keywords Potential theory · Approximation - Complex function theory

\section{A few words about Stephen}

Stephen Gardiner was born in 1958 in Belfast, where he pursued his undergraduate and graduate studies in Mathematics. In 1982 he obtained his $\mathrm{PhD}$ from Queen's University under the supervision of David Armitage. Following his doctoral thesis on Generalized Means of Subharmonic Functions, Gardiner worked on a wide range of topics on potential theory and became one of the leading experts in this area. In 1995 he was awarded the degree of Doctor of Science for his published work on Approximation, Boundary Behaviour and Convexity in Potential Theory.

$\triangle$ Hermann Render

hermann.render@ucd.ie

Marius Ghergu

marius.ghergu@ucd.ie

Myrto Manolaki

arhimidis8@yahoo.gr

Ivan Netuka

netuka@karlin.mff.cuni.cz

1 University College Dublin, Belfield 4, Dublin, Ireland

2 Faculty of Mathematics and Physics, Mathematical Institute of Charles University, Sokolovská 83, 18675 Praha 8, Czech Republic

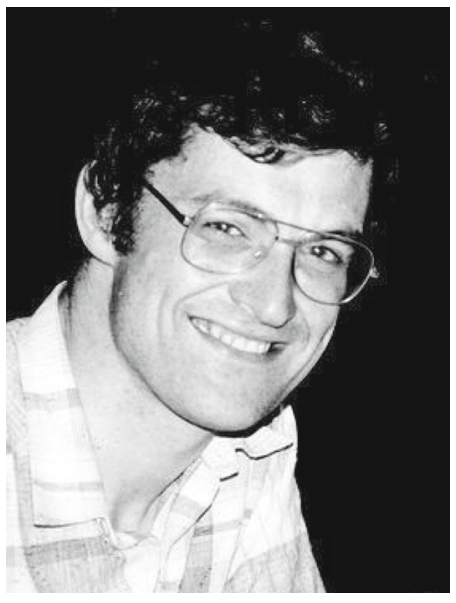

Stephen in 1987 
In 1984 Stephen joined University College Dublin, where he is currently Professor of Mathematics (the eighth holder of the original chair since the foundation of the university in 1854). In addition to an impressive research career, he has given tireless academic service in various roles in Ireland and internationally. In UCD he has served as Associate Dean in the Faculty of Arts, as Head of the Department of Mathematics and since 2007 he has been the School Head of Finance and Staffing. Moreover, in 2000 he was elected a Member of the Royal Irish Academy (the highest academic honour in Ireland) and in 2018 he was further elected to the office of Treasurer of the Academy.

At an international level, he served as the Irish co-ordinator of the European Union Research Training Network "Classical Analysis, Operator Theory, Geometry of Banach Spaces, their Interplay and Applications" (2000-2004) and as a member of the Steering Committee of the European Science Foundation Research Network "Harmonic and Complex Analysis and its Applications" (2007-2012). He also served as an Editorial Adviser for the Proceedings, Journal and Bulletin of the London Mathematical Society and is currently on the Editorial Board of Potential Analysis.

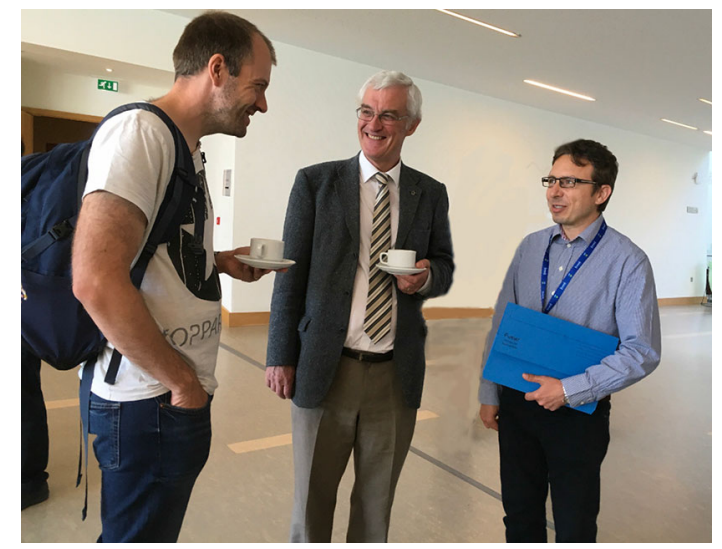

Stephen at the conference ICCAPTA 2018 with his former postdocs Tomas Sjödin and Marius Ghergu

Stephen is a charismatic lecturer, and an insightful academic leader. He has been the Principal Investigator of several research projects, and supervised 4 doctoral students and 5 postdoctoral fellows. His mathematical work, which is characterized by depth and elegance, has been recognized internationally. He has 21 collaborators and has delivered numerous invited lectures around the world. His research record includes 113 papers on various aspects of potential theory, complex function theory and approximation and two important monographs:

B1. Harmonic approximation, London Math. Soc. Lecture Note Series 221, Cambridge University Press, 1995, xiv + 132pp.

B2. Classical potential theory, Springer Monographs in Mathematics, Springer, London, 2001, xvi + 333pp (with D. H. Armitage). 
The monograph $\mathrm{B} 1$ is the first book to give a systematic account of the developments of harmonic approximation. It took shape in 1992, when Gardiner spent his sabbatical leave at McGill University in Canada, during which he managed to solve some of the main open problems of the area.

His second monograph B2, jointly written with David Armitage, provides a comprehensive treatment of classical potential theory and is considered one of the standard references of the field (it has

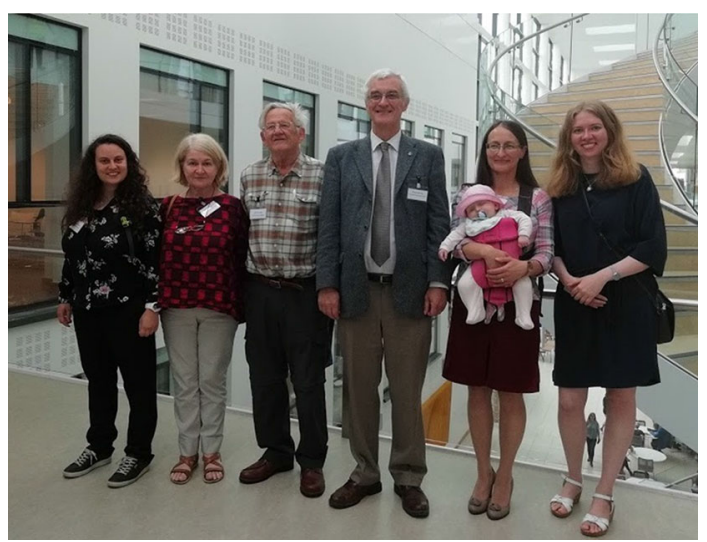

Stephen with his doctoral students (from left to right, Myrto Manolaki, Mary Hanley, Joanna Pres and Mayya Golitsyna) and supervisor David Armitage received more than 300 citations). As Matts Essén wrote in Math Reviews it is an excellent addition to the mathematical literature.

As we shall demonstrate, Gardiner's contributions to Mathematics are deep, with broad impact in several areas of Mathematical Analysis. We focus on his main mathematical achievements in the following diverse topics:

1. The exterior inverse problem in potential theory

2. Quadrature domains

3. Stationary points of Hele-Shaw flows

4. Fine potential theory

5. Harmonic approximation

6. Universal Taylor series and potential theory

7. Potential theory on cylindrical sets

8. Analytic content

We have included the scientific bibliography of Stephen Gardiner at the end of this survey and, for simplicity, articles (including those with co-authors) will be cited as [Gx] for $x=1, \ldots, 113$.

\section{The exterior inverse problem of potential theory}

We denote a typical point in the Euclidean space $\mathbb{R}^{n}$ by $x$ or $\left(x_{1}, \ldots, x_{n}\right)$ and write

$$
\|x\|=\sqrt{x_{1}^{2}+\cdots+x_{n}^{2}}
$$


The Newtonian potential of a positive Radon measure $\mu$ with compact support in $\mathbb{R}^{n}$ is a fundamental notion in Potential Theory, see [B2]. For $n \geq 3$ it is defined by

$$
U^{\mu}(x)=\int_{\mathbb{R}^{n}}\|x-y\|^{2-n} d \mu(y)
$$

When $n=2$ the Newtonian kernel $\|x-y\|^{2-n}$ is replaced by the logarithmic kernel $-\ln \|x-y\|$. If $\mu$ is the restriction of the volume measure $\lambda$ to a bounded Borel set $A$, we will write

$$
U^{A}=U^{\lambda \mid A}
$$

Let us recall that a bounded domain $\Omega$ in Euclidean space $\mathbb{R}^{n}$ is called solid if the complement of the closure of $\Omega$ is connected and the interior of the closure of $\Omega$ is equal to $\Omega$. A long-standing open question, known as the exterior inverse problem of potential theory, asks:

If $\Omega_{1}$ and $\Omega_{2}$ are two solid domains in $\mathbb{R}^{n}$ such that $U^{\Omega_{1}}=U^{\Omega_{2}}$ on $\left(\Omega_{1} \cup \Omega_{2}\right)^{c}$, does it follow that $\Omega_{1}=\Omega_{2}$ ?

If we drop the requirement that $\Omega_{1}$ and $\Omega_{2}$ are solid domains, then the answer to the above question is "no"; one can simply consider a ball and a suitably chosen concentric annular domain of equal measure. Less trivial examples (e.g. [36,44]) show that the answer would again be "no" if the equality $U^{\Omega_{1}}=U^{\Omega_{2}}$ were required to hold only in a neighbourhood of infinity or, equivalently, throughout the unbounded component of $\left(\overline{\Omega_{1} \cup \Omega_{2}}\right)^{c}$.

Novikov showed in 1938 that, if we require both $\Omega_{1}$ and $\Omega_{2}$ to be starlike with respect to a common point (in particular, if they are convex), then the answer is "yes". Kondraškov [27] proved that the answer to the question is also "yes" if one of the domains is a ball or an ellipsoid (see [2]); an elegant elementary proof for the case of a ball may be found in [44]. Further, Shahgholian [43] proved that the answer to the above question is affirmative if one requires only that $\Omega_{1} \cap \Omega_{2}$ is convex.

Using partial balayage techniques, Stephen Gardiner and Tomas Sjödin proved in [G84] the following beautiful result:

Theorem 1 Let $\Omega_{1}$ be a solid domain and $\Omega_{2}$ be a convex domain, and let $v$ be a measure such that $v \geq\left.\lambda\right|_{\Omega_{2}}$ and $v\left(\Omega_{2}^{c}\right)=0$. If $U^{\Omega_{1}}=U^{v}$ on $\left(\Omega_{1} \cup \Omega_{2}\right)^{c}$, then $\Omega_{2} \subseteq \Omega_{1}$.

The particular case of the above theorem where $v=\left.\lambda\right|_{\Omega_{2}}$ shows that the answer to the above question is affirmative if one merely assumes one of the domains to be convex. 


\section{Quadrature domains}

A bounded domain $\Omega$ in Euclidean space $\mathbb{R}^{n}(n \geq 2)$ is called a quadrature ${ }^{1}$ domain (for harmonic functions) if there is a signed Borel measure $\mu$, with compact support in $\Omega$, such that the following integral identity

$$
\int_{\Omega} h d \lambda=\int h d \mu,
$$

holds for every integrable harmonic function $h$ on $\Omega$ where $\lambda$ denotes the Lebesgue measure. We then say that $\Omega$ is a quadrature domain with respect to $\mu$.

Using the classical mean value property for harmonic functions, it is easy to see that any open ball $B$ is a quadrature domain if we choose $\mu$ to be the point measure concentrated at the centre of $B$ with mass $\lambda(B)$. However, there are many other possible choices of signed measure $\mu$ here, and many examples of quadrature domains other than balls. For instance, (1) holds for an ellipse if we choose $\mu$ to be a suitably weighted form of one-dimensional measure on the line segment joining its foci (see, for example, [41]). Quadrature domains arise naturally, not only in potential theory, but also in several other areas of mathematical science; one such example is the Hele-Shaw moving boundary problem.

As is well known, positive harmonic functions need not be integrable on bounded domains: for example, the harmonic function $x_{1} x_{2}\|x\|^{-4}, x \in \mathbb{R}^{2}$, is clearly not integrable on the square $(0,1)^{2}$. In contrast, Gardiner and Sjödin established in [G80] the following result:

Theorem 2 Every positive harmonic function on a quadrature domain $\Omega$ is integrable on $\Omega$.

The proof of Theorem 2 relies on local estimates for free boundary problems (see [8]) and properties of the Martin kernel.

A significant question concerning the definition of quadrature domains is whether a quadrature domain with respect to a signed measure is also a quadrature domain with respect to some positive measure.

In the case where $n=2$, Gustafsson, Sakai and Shapiro [21] gave a positive answer to this question. The approach in [21] combines conformal mappings with deep results of Sakai [38,39], concerning the geometrical classification of plane quadrature domains, which show that any such quadrature domain contains a finitely connected quadrature domain.

This approach is clearly not available in higher dimensions. It was nevertheless conjectured in [21] that the answer is "yes" in any dimension.

From Theorem 2, through a limiting process, Gardiner and Sjödin deduced in [G80]:

Theorem 3 If $\Omega$ is a quadrature domain with respect to a signed measure $\mu$ with compact support in $\Omega$, then it is also a quadrature domain with respect to some positive measure with compact support in $\Omega$.

\footnotetext{
1 The word "quadrature" has Latin origin meaning "making square-shaped". It appeared in mathematics in the middle of 16th century in connection with constructive or numerical methods for determining areas, and more recently with computing integrals in general.
} 
Further results on quadrature domains may be found in the papers [G94] and [G99].

\section{Stationary points of Hele-Shaw flows}

Hele-Shaw flow is defined as Stokes flow between two parallel flat plates separated by an infinitesimally small gap, named after H. S. Hele-Shaw, who studied the problem in 1898. Various problems in fluid mechanics can be approximated by Hele-Shaw flows and thus the research of these flows is important.

In this section we consider the problem for higher dimenisons and we assume that a slow, viscous, incompressible fluid is trapped in a region $\Omega_{0} \subset \mathbb{R}^{n}(n \geq 2)$ between two close parallel hyperplanes. Fluid is then injected through a fixed point $p \in \Omega_{0}$ at time $t=0$ and one wants to investigate the evolution of the domain $\Omega_{t}$ of the fluid at time $t$.

It is a classical fact that the domain $\Omega_{t}$ can be characterized as the smallest domain $\Omega$ containing $\Omega_{0}$ such that

$$
\int_{\Omega} s d \lambda \geq \int_{\Omega_{0}} s d \lambda+t \cdot s(p)
$$

for all Lebesgue-integrable subharmonic functions $s$ on $\Omega$. Indeed, M. Sakai [37] provided a proof that such domains $\Omega$ exist and are unique up to $\lambda$-null sets, and that there is a smallest one, which we will denote in the following by $\Omega_{t}$. This fact also underlines the importance of the notion of a quadrature domain and its obvious generalization to the class of subharmonic functions, which is relevant to the HeleShaw flow.

A fascinating problem concerning Hele-Shaw flow is to understand the qualitative behaviour of the domain $\Omega_{t}$ as the time $t$ evolves. A point $q \in \partial \Omega_{0}$ is called (initially) stationary for the family $\left\{\Omega_{t}: t \geq 0\right\}$ if there exists $\varepsilon>0$ such that $q \in \partial \Omega_{t}$ whenever $0<t<\varepsilon$. In dimension $n=2$ it has been shown (see Sakai [40] and earlier work of King et al. [26]) that, if the boundary of the domain $\Omega_{0}$ has a corner $q$ with interior angle less than $\pi / 2$, then this point is (initially) stationary. Further, corners of angle greater than $\pi / 2$ are not stationary, and corners of angle $\pi / 2$ may or may not be stationary.

One remarkable achievement of Stephen Gardiner and Tomas Sjödin is the discovery of analogous results in higher dimensions in [G101]. We need the following notations and definitions: let $B(x, r)$ denote the open ball in $\mathbb{R}^{n}$ of centre $x$ and radius $r>0$, and let $S(x, r)=\partial B(x, r)$. For any non-empty relatively open subset $\omega$ of the unit sphere $S:=S(0,1)$ we define

$$
\ell(\omega)=\inf \left\{\frac{\int_{S}\left|\nabla_{S} f\right|^{2} d \sigma}{\int_{S} f^{2} d \sigma}: f \in L^{+}(S) \text { and } f(x)=0 \text { for all } x \in S \backslash \omega\right\},
$$

where $L^{+}(S)$ is defined as the set of all non-zero, non-negative Lipschitz functions on $S$. It is known that, for a connected set $\omega$, the quantity $\ell(\omega)$ is the first eigenvalue 
of the Laplace-Beltrami operator $-\Delta_{S}$. The characteristic constant of a set $\omega \subset S$ is defined as the nonnegative root of the quadratic equation

$$
\alpha(\alpha+n-2)=\ell(\omega) .
$$

Note that, if $n=2$ and $\omega$ is an arc of length $\theta$, then $\alpha(\omega)=\pi / \theta$.

If $L$ is a compact subset of $S$ we define

$$
\alpha(L)=\sup \{\alpha(\omega): \omega \text { is relatively open in } S \text { and } L \subset \omega\}
$$

Next we describe a criterion for when a point $q \in \partial \Omega_{0}$ is initially stationary for the family $\left\{\Omega_{t}: t \geq 0\right\}$. By a simple translation argument we may assume that $q=0 \in \partial \Omega_{0}$. In the first result we shall assume that there exists $r_{0}>0$ and a compact subset $L$ of the unit sphere $S$ such that

$$
\Omega_{0} \cap B\left(0, r_{0}\right) \subset K(L):=\{r y: r>0, y \in L\} .
$$

The set $K(L)$ is called the conical set of $L$ and the last condition is a convenient way to require special properties of the boundary point $q=0$ in higher dimensions.

Theorem 4 Let $L$ be a compact subset of $S$ such that $\alpha(L)>2$, and suppose there exists $r_{0}>0$ such that $\Omega_{0} \cap B\left(0, r_{0}\right) \subset K(L)$. Then 0 is initially stationary for $\left\{\Omega_{t}: t \geq 0\right\}$.

The next result shows that the assumption $\alpha(L)>2$ is crucial in Theorem 4 .

Theorem 5 Let $\omega$ be a domain relative to $S$, with $C^{1, \beta}$ boundary.

(a) If $\alpha(\omega) \leq 2$ and $K(\omega) \cap B\left(0, r_{0}\right) \subset \Omega_{0}$ for some $r_{0}>0$, then 0 is not initially stationary for $\left\{\Omega_{t}: t \geq 0\right\}$.

(b) Further, if $\alpha(\omega)=2$ and $\psi:(0,1] \rightarrow(0,1 / 2]$ is increasing and satisfies $\int_{0}^{1} t^{-1} \psi(t) d t<\infty$, then 0 is not initially stationary for $\left\{\Omega_{t}: t \geq 0\right\}$, where

$$
\Omega_{0}=\left\{x \in B(0,1): \operatorname{dist}\left(x, K(\omega)^{c}\right)>\|x\| \psi(\|x\|)\right\} .
$$

The following result shows that in the case $\alpha(\omega)=2$ boundary points might also be stationary:

Theorem 6 Let $\omega$ be a domain relative to $S$, with Lipschitz, boundary, such that $\alpha(\omega)=$ 2. Then there is a constant $C(\omega)>1$ such that 0 is initially stationary for $\left\{\Omega_{t}: t \geq 0\right\}$, where

$$
\Omega_{0}=\left\{x \in B\left(0, e^{-2 C(\omega)}\right): \operatorname{dist}\left(x, K(\omega)^{c}\right)>\frac{C(\omega)\|x\|}{\log (1 /\|x\|)}\right\} .
$$

For any increasing continuous function $\phi:(0, \infty) \rightarrow(0,1 / 2]$ satisfying the doubling condition $\phi(2 t)<C \phi(t)$ for some $C>1$, we define the enlarged domain

$$
\Omega_{0}(\phi)=\left\{x \in \mathbb{R}^{n} \backslash\{0\}: \operatorname{dist}\left(x, \Omega_{0}\right)<\|x\| \phi(\|x\|)\right\}
$$

which has $q=0$ as a boundary point. Some of the above cited results were derived in [G101] from the following: 
Theorem 7 Let $\phi:(0, \infty) \rightarrow(0,1 / 2]$ be an increasing continuous function satisfying the doubling condition and let $p_{0} \in \Omega_{0}$ such that

$$
\varphi(\rho) \geq C_{0} \exp \left\{\frac{1}{n} \int_{\rho}^{\left|p_{0}\right|} \frac{2-\alpha\left(\Omega_{0}(\phi) \cap S(0, t)\right)}{t} d t\right\}\left(0<\rho<\left|p_{0}\right|\right)
$$

for some positive constant $C_{0}$. Then there exists $\varepsilon>0$ such that $\Omega_{t} \subset \Omega_{0}(\phi)$ when $0<t<\varepsilon$. In particular, 0 is initially stationary for $\left\{\Omega_{t}: t \geq 0\right\}$.

The following example illustrates the results: Let $0<\theta_{0}<\pi / 2$. It follows from the above results that the origin is initially stationary for the truncated cone

$$
\Omega_{0}=\left\{\left(x_{1}, \ldots, x_{n}\right) \in B(0,2): x_{n}>\left(\cos \theta_{0}\right)\|x\|\right\},
$$

if and only if $\theta_{0}<\cos ^{-1}(1 / \sqrt{n})$.

Also, given $0<\theta_{0}<\pi$, the point 0 is initially stationary for the truncated wedge

$$
\Omega_{0}=\left\{\left(x_{1}, \ldots, x_{n-2}, r \cos \theta, r \sin \theta\right): 0<r<2,0<\theta<\theta_{0}\right\}
$$

if and only if $0<\theta_{0}<\pi / 2$.

\section{Fine potential theory}

The fine topology has an intimate relationship to the Dirichlet problem. Its roots go back to the notions of irregular and unstable boundary points of a domain and a compact set in $\mathbb{R}^{n}$, respectively.

Let us note that at the beginning of the twentieth century, it was generally believed that the Dirichlet problem on a bounded domain $U \subset \mathbb{R}^{n}$ was solvable for all continuous boundary conditions. Lebesgue's counterexample from 1913 showed that it was not the case. Lebesgue's result constituted a breakthrough for the understanding of exceptional boundary points and reveals the importance of discontinuous potentials. We briefly recall Lebesgue's idea. Let $\mu$ be a measure on the line segment

$$
I=\left\{(t, 0,0) \in \mathbb{R}^{3}: 0 \leq t \leq 1\right\}
$$

with density $t$ at $(t, 0,0)$. Let $p$ be the Newtonian potential of $\mu$, that is

$$
p(x)=\int_{I \backslash\{0\}} \frac{d \mu(y)}{\|x-y\|} \quad \text { for } x \in \mathbb{R}^{3} .
$$

Then $p(0)=1$ and $p(x)=\infty$ for $x \in I \backslash\{0\}$. Hence the potential $p$ is discontinuous, and the intersection $U$ of the unit ball with the set $\left\{x \in \mathbb{R}^{3}: p(x)<2\right\}$ is simply connected. The point 0 is a non-isolated boundary point of $U$, which is irregular for the Dirichlet problem on $U$. In fact, the complement of $U$ has a very sharp thorn at 0 (the Lebesgue spine). 
This result triggered a series of investigations on the potential theoretic classification of boundary points. As shown by N. Wiener in 1924, an irregular point $x \in \partial U$ is characterized by the complement $E$ of $U$ being thin at $x$ in the sense that

$$
\sum_{j=1}^{\infty} \frac{\operatorname{cap}^{*}\left(E \cap B_{j}\right)}{\operatorname{cap}^{*}\left(B_{j}\right)}<\infty ;
$$

here cap* stands for the outer capacity and $B_{j}$ is the ball of radius $2^{-j}$ centered at $x$.

The condition (4) could be taken as the definition of thinness for an arbitrary set $E \subset \mathbb{R}^{n}$. However, for further developments of potential theory, an equivalent definition of thinness introduced by M. Brelot in 1939 turned out to be extremely useful. The analogy of criteria for regular and stable points for the Dirichlet problem led M. Brelot to the following definition: a set $E$ in $\mathbb{R}^{n}$ is called thin (effile in French) at $x$ if either $x \notin \bar{E}$ or

$$
u(x)<\liminf _{y \rightarrow x, y \in E \backslash\{x\}} u(y)
$$

for some superharmonic function $u$ defined in a neighbourhood of $x$. Using this notion, $x \in \partial U$ is irregular if and only if $\mathbb{R}^{n} \backslash U$ is thin at $x$. Similarly, for a compact set $K$ in $\mathbb{R}^{n}$, $x \in \partial K$ is unstable, if and only if $\mathbb{R}^{n} \backslash K$ is thin at $x$.

In Mathematics, it happens only rarely that a precise birthday is known for a mathematical notion. ${ }^{2}$ In a letter of the 30th December 1940 addressed to M. Brelot, H. Cartan pointed out that, for a point $x \in \mathbb{R}^{n}$, the system of all complements of sets $E \backslash\{x\}$, where $E$ is thin at $x$, forms a local base for a topology, later called by him the fine topology. In fact, the fine topology is the coarsest topology on $\mathbb{R}^{n}$ for which all superharmonic functions on open subsets of $\mathbb{R}^{n}$ are continuous. For quite some time analysts considered the fine topology somewhat artificial, pathological and difficult to handle. Although Hausdorff, completely regular and Baire, it is not normal, does not satisfy the axioms of countability, nor has the Lindelöf property. On top of that, the only finely compact sets are finite sets. For probabilists, however, the fine topology is very natural: a Borel set $V$ in $\mathbb{R}^{n}$ is finely open if and only if a Brownian particle, starting at a point of $V$, remains in $V$ almost surely in some positive time interval.

Gradually, useful properties of the fine topology were found:

1. The fine topology has the quasi-Lindelöf property, that is, an arbitrary union of finely open subsets of $\mathbb{R}^{n}$ differs by a polar set from some countable subunion (J. L. Doob, 1954).

2. Finely closed sets differ from suitable closed subsets by sets of arbitrarily small capacity (G. Choquet, 1959).

3. The fine topology is locally connected (B. Fuglede, 1969).

4. Every finely continuous function is a Baire-1 function (B. Fuglede, 1974).

5. The Lusin-Menchoff property (J. Lukeš, 1977).

6. Every fine domain is polygonally connected (T.J. Lyons, 1980).

2 As a notable example we mention the famous quaternion plaque on Brougham (Broom) Bridge in Dublin which says: "Here as he walked by on the 16th October 1843 Sir William Rowan Hamilton in a flash of genius discovered the formula for quaternion multiplication $i^{2}=j^{2}=k^{2}=i j k=-1$, and cut it on the stone of the bridge". 
Since the 1970s elegant theories of harmonic as well as holomorphic functions with respect to fine topologies have been developed, also in a general potential theoretic setting. For more information and relevant bibliography we refer the reader to [B2, Chap. 7], [7, 14, 15, 28]. Several papers of Stephen Gardiner are related to the fine topology: [G23], [G28], [G60], [G65], [G67], [G75], [G81], [G83], [G90], [G93] and [G96]. Due to space limitation we present only some of his main results, in particular those providing solutions to longstanding open problems.

We first recall the following fundamental result in classical potential theory: Let $V$ be a domain in $\mathbb{R}^{n}$ (with non-polar complement when $n=2$ ) and let $G_{V}$ be the Green function for $V$. The decomposition theorem of F. Riesz (1930) tells us that the following conditions are equivalent for a non-negative superharmonic function $u$ on $V$ :

(i) The only non-negative harmonic minorant of $u$ is zero.

(ii) There exists a measure $\mu$ on $V$ such that $u(x)=\int G_{V}(x, y) d \mu(y)$ for $x \in V$.

Finely harmonic and finely superharmonic functions can be defined in a natural way in the setting of fine potential theory using swept-out measures leading also to the new concept of the fine Green function.

In 1972, B. Fuglede raised the question whether the fine topology counterparts of conditions (i) and (ii) above are also equivalent, see [14, p. 105]. The importance of this problem is emphasized in [15, Problem P2]. In 2007, Stephen Gardiner and Wolfhard Hansen (see [G81]) answered the question in the negative for $n \geq 3$ (the case $n=2$ is still open!). The proof is intricate and is based on delicate results concerning minimal harmonic functions associated with irregular boundary points and minimal thinness.

Let us discuss another natural problem: Given an open set $U$ in $\mathbb{R}^{n}$, is fine harmonicity equivalent to harmonicity on $U$ ? Fuglede showed that this is the case for $n=2$. For a finely harmonic function $u$ defined on an open set $U$ in $\mathbb{R}^{n}$ with $n \geq 2$ he proved that there is a dense open subset $V$ of $U$ on which $u$ is harmonic and showed that $U \backslash V$ may be non-empty if $n \geq 3$. In [G75], Stephen Gardiner established the sharpness of Fuglede's theorem by proving the following:

Theorem 8 Let $V \subset U$ be open subsets in $\mathbb{R}^{n}$. Then $V$ is dense in $U$ if and only if there exists a finely harmonic function $u$ on $U$ such that $V$ is the largest open subset of $U$ on which $u$ is harmonic.

In [14, p. 75] the following question was raised: Given a finely open set $U$ without interior points in the Euclidean topology, does there exist a finely harmonic function $u$ on $U$ such that no restriction to a non-empty finely open subset of $U$ admits a harmonic extension? In [G93] Stephen Gardiner proved the following:

Theorem 9 Let $U$ be a non-empty finely open set in $\mathbb{R}^{n}$. Then the following statements are equivalent:

(i) for any finely harmonic function $u$ on $U$ there is a non-empty finely open subset $V$ of $U$ and a harmonic function $w$ defined on an open set $W$ containing $V$ such that $w=u$ on $V$.

(ii) for any finely harmonic function $u$ on $U$ there is a harmonic function $w$ on an open set $W$ such that $W \backslash U$ is polar and $w=u$ on $U \cap W$. 
(iii) $U$ is of second Baire category with respect to Euclidean topology.

So, defining $U=B \backslash Z$ where $B$ is an open ball and $Z$ is a countable dense subset of $B$, we obtain a counterexample to Fuglede's question.

It is a matter of fact that many problems in potential theory can only be solved by using the fine topology. We illustrate this by considering the set $\mathcal{D}$ of all harmonic functions $h$ defined on the unit ball $B$ in $\mathbb{R}^{n}$ with finite Dirichlet integral, that is

$$
\int_{B}|\nabla h(x)|^{2} d x<\infty .
$$

One is interested in characterizing subsets $E$ of $B$ such that

$$
\sup h(E)=\sup h(B) \quad \text { for all } h \in \mathcal{D} \text {. }
$$

Let us denote by $E_{N T}$ the set of points of $\partial B$ which are non-tangential limits of a sequence in $E$. Stephen Gardiner established the following nice result in [G65]:

Theorem 10 For a subset $E$ of the unit ball $B$ in $\mathbb{R}^{n}$ the equality (5) holds if and only if $E_{N T}$ is finely dense in $\partial B$.

We conclude this section with a discussion about fine differentiability. Let $U$ be a finely open set and $f: U \rightarrow \mathbb{R}$ be a function. A linear mapping $L_{z}: \mathbb{R}^{n} \rightarrow \mathbb{R}$ is called the fine differential of $f$ at the point $z \in U$ if the quotient

$$
\frac{f(x)-f(z)-L_{z}(x-z)}{\|x-z\|}
$$

has the limit 0 when $x$ approaches $z$ in the fine topology. A function $f: U \rightarrow \mathbb{R}$ is called finely continuously differentiable if the fine differential $L_{z}$ exists for every $z \in U$ and the mapping $z \longmapsto L_{z}$ is finely continuous on $U$. A sufficient condition for this property is finely local continuous differentiability: that is, for each $z \in U$ there exists a fine neighbourhood $V$ of $z$ and a function $\tilde{f} \in C^{1}\left(\mathbb{R}^{n}\right)$ such that $f$ is equal to $\widetilde{f}$ on $V$. In [G90] Stephen Gardiner proved

Theorem 11 Every finely continuously differentiable function $f: U \rightarrow \mathbb{R}$ is finely locally continuously differentiable.

Let us note that this result was proven by R. Lávička for dimension $n=2$ already in 2007. He pointed out that, in higher dimensions, it is not even clear whether a function that has zero fine differential at every point of a finely connected finely open set must be constant. That this is indeed the case follows from Theorem 11 and the fact that the fine topology is locally connected and polygonally connected.

\section{Harmonic approximation}

During the period 1993-2001, Stephen Gardiner published a series of significant results about approximation in potential theory: [G37], [G39], [G40], [G41], [G42], 
[G43], [G44], [G47], [G48], [G51], [G53], [G54], [G57], [G70], and [G71]. The monograph [B1] gives a nicely written account of the theory of harmonic approximation in Euclidean space $\mathbb{R}^{n}, n \geq 2$. The exposition of various aspects, as well as applications, of this type of approximation can be found in the survey papers [G38], [G46], [G52], [G59] and [G66].

An early result on harmonic approximation in the higher dimensional case is due to J.L. Walsh: If $K$ is a compact set and $\mathbb{R}^{n} \backslash K$ is connected, then every function harmonic near $K$ can be approximated uniformly on $K$ by functions that are harmonic on the whole $\mathbb{R}^{n}$ (and hence by harmonic polynomials). The connectedness of $\mathbb{R}^{n} \backslash K$ is not, unlike in the case of holomorphic approximation, a necessary condition. The situation of compact sets with holes, however, requires more delicate notions from potential theory. An even more complicated situation occurs if we are interested in harmonic approximation on general relatively closed subsets of an open set in $\mathbb{R}^{n}$.

In order to present a sample of Gardiner's results we shall introduce the following notations and definitions:

Let $U$ be a connected open subset of $\mathbb{R}^{n}$ and $E$ be a relatively closed subset of $U$. By $\mathcal{H}(E)$ and $\mathcal{S}(E)$ respectively we denote the set of all functions which are harmonic (superharmonic, respectively) on some open set containing $E$.

A subset $A$ of $U$ is called $U$-bounded if the closure $\bar{A}$ is a compact subset of $U$. We denote by $\widehat{E}$ the union of $E$ with all the $U$-bounded components of $U \backslash E$. We will say that the pair $(U, E)$ satisfies the $(K, L)$-condition if for each compact subset $K$ of $U$ there is a compact subset $L$ of $U$ which contains every $U$-bounded component of $U \backslash(E \cup K)$ whose closure intersects $K$.

Now we can present the following Runge-type results established by Gardiner in [G37], see also p. 51 in [B1].

Theorem 12 The following statements are equivalent:

(i) For each $u$ in $\mathcal{H}(E)$ and each positive number $\varepsilon$ there exists $v \in \mathcal{H}(U)$ such that $|v-u|<\varepsilon$ on $E$.

(ii) For each $u \in \mathcal{H}(E)$ and each strictly positive $s$ in $\mathcal{S}(E)$ there exists $v \in \mathcal{H}(U)$ such that $0<v-u<s$ on $E$.

(iii) $U \backslash \widehat{E}$ and $U \backslash E$ are thin at the same points of $E$ and $(U, E)$ satisfies the $(K, L)$ -condition.

Combining this theorem with known results, Stephen Gardiner established in [G37] (see also p. 57 in [B1]) the harmonic analogue of Arakelyan's generalization of Mergelyan's Theorem to the non-compact case:

Theorem 13 The following statements are equivalent:

(i) For each function $u$ which is continuous on $E$ and harmonic on the interior $E^{\circ}$ of $E$ and for each positive number $\varepsilon$ there is a harmonic function $v$ on $U$ such that $|v-u|<\varepsilon$ on $E$.

(ii) $U \backslash \widehat{E}$ and $U \backslash E^{\circ}$ are thin at the same points of $E$ and $(U, E)$ satisfies the $(K, L)$ condition.

In the previous theorem, what can be said about the speed of approximation at infinity? To give an answer, we introduce the following notion: we will say that the 
pair $(U, E)$ satisfies the long islands condition if for each compact subset $K$ of $U$ there is a compact subset $L$ of $U$ which contains every component of $E^{\circ}$ that intersects $K$.

The harmonic analogue of Nersesyan's result on Carleman-type holomomorphic approximation, established in [G41] and [G42], reads as follows:

Theorem 14 The following statements are equivalent:

(i) For each function $u$ which is continuous on $E$ and harmonic on $E^{\circ}$ and for each continuous function $\varepsilon: E \rightarrow(0,1]$ there is a harmonic function $v$ on $U$ such that $|v-u|<\varepsilon$ on $E$.

(ii) $U \backslash \widehat{E}$ and $U \backslash E^{\circ}$ are thin at the same points of $E$ and $(U, E)$ satisfies both the $(K, L)$-condition and the long islands condition.

In a moment we shall discuss two problems where harmonic approximation on non-compact sets turns out to be a useful tool. Before that, we mention one result on superharmonic extension proved in [G37]. In this result $U^{*}$ denotes the Alexandroff compactification of $U$.

Theorem 15 The following statements are equivalent:

(i) For every function $s \in \mathcal{S}(E)$ there exists a superharmonic function $v$ on $U$ such that $v=s$ on an open set which contains $E$.

(ii) $U^{*} \backslash E$ is connected and locally connected.

The first application of harmonic approximation deals with the Dirichlet problem. Let $U$ be an open set in $\mathbb{R}^{n}$. We denote by $\partial_{r} U$ and $\partial_{i} U$ respectively the set of all regular and irregular points respectively. We know that $x \in \partial_{i} U$ is equivalent to the statement that $\mathbb{R}^{n} \backslash U$ is thin at $x$. If $U$ is bounded and $f$ is a continuous function on the boundary $\partial U$, then the Perron-Wiener-Brelot method provides a (unique) harmonic function $h_{f}$ on $U$ such that

$$
\begin{aligned}
& \text { for } z \in \partial_{r} U \text { we have } \lim _{x \rightarrow z} h_{f}(x)=f(z), \\
& \text { for } z \in \partial_{i} U \text { we have } \limsup _{x \rightarrow z}\left|h_{f}(x)\right|<\infty .
\end{aligned}
$$

However, if $U$ is unbounded then it is not obvious that a harmonic function $h_{f}$ satisfying both conditions exists. Using a harmonic approximation technique, Stephen Gardiner gave in [G33] the following characterization of sets admitting a solution of the Dirichlet problem:

Theorem 16 The following statements are equivalent for a domain $U$ :

(i) For every continuous function $f$ on $\partial U$ there exists a harmonic function $h_{f}$ on $U$ which satisfies (6) and (7).

(ii) $\left(\mathbb{R}^{n}, \mathbb{R}^{n} \backslash U\right)$ satisfies the $(K, L)$-condition.

As a corollary, the following strong non-uniqueness result follows: If $U$ is an unbounded domain and if for each continuous function $f$ on $\partial U$ there exists a harmonic function $h_{f}$ on $U$ which satisfies (6) and (7), then for each continuous function 
$f$ on $\partial U$ there exist infinitely many distinct harmonic functions $h_{f}$ on $U$ which satisfy (6) and (7).

The concluding result in this section, proved by Gardiner and Hansen in [G67] using an approximation result for finely harmonic functions, gives a solution of an interesting problem related to boundary behaviour of harmonic functions: Let $D$ denote the upper half-space $\left\{(x, t): x \in \mathbb{R}^{n}, t>0\right\}$ in $\mathbb{R}^{n+1}$ and let $\lambda$ denote the Lebesgue measure on $\mathbb{R}^{n}$. Then it is asked for which sets $E$ in $\mathbb{R}^{n}$ is there a harmonic function $u$ on $D$ such that

$$
\lim _{t \rightarrow 0+} u(x, t)=\infty \text { for all } x \in E ?
$$

For $n=1$, the problem has a holomorphic counterpart that was solved long ago; for an overview of relevant results we refer to [G66, pp. 212-213]. Moreover, a harmonic function $u$ satisfying (8) exists if and only if for each interval $I$ the set $E \cap I$ is of first category or there is some non-empty subinterval $J$ of $I$ such that $\lambda(E \cap J)=0$.

A higher-dimensional analgoue of the last statement, replacing interval by nonempty open set in $\mathbb{R}^{n}$, fails. In fact, both the Euclidean and fine topologies are needed for the following characterization proved in [G67] (note that for the case $n=1$ the fine topology coincides with the Euclidean topology):

Theorem 17 Let $E$ be a set in $\mathbb{R}^{n}$. Then the following statements are equivalent:

(i) There exists a harmonic function $u$ on the upper half-space $D$ in $\mathbb{R}^{n+1}$ which satisfies (8).

(ii) There exists a continuous superharmonic function u on D satisfying (8).

(iii) There is an increasing sequence $\left(E_{k}\right)$ of sets such that

$$
E=\bigcup_{k=1}^{\infty} E_{k} \text { and } \lambda\left(E \cap V_{k}\right)=0
$$

for each $k \in \mathbb{N}$ where $V_{k}$ denotes the fine interior of $\overline{E_{k}}$.

\section{Universal Taylor series and potential theory}

The main idea of universality is that a single object, via a countable process, can approximate a universal class of objects. The phenomenon of universality was first observed by Fekete [35] in 1914. He showed that there exists a real power series $\sum_{n=1}^{\infty} a_{n} x^{n}$ having radius of convergence 0 , with the following property: for each continuous function $g:[-1,1] \rightarrow \mathbb{R}$ with $g(0)=0$ there is a subsequence of partial sums that converges to $g$ uniformly on $[-1,1]$. After Fekete's result, several universal objects were discovered. For example, Birkhoff [6] proved the existence of an entire function with universal translations, and later MacLane [29] showed the existence of an entire function with universal derivatives. The common feature in these two examples is that the approximation is obtained by iterations of a single continuous linear operator (the translation and differentiation operators, respectively). This type of universality, known as hypercyclicity, has been studied intensively and is a rapidly developing research area: see [17] for an account up to 1999. 
Stephen Gardiner wrote a series of papers [G89], [G95], [G97], [G98], [G100], [G102], [G103], [G106], [G107] on another widely-studied instance of universality: the case of universal Taylor series of holomorphic functions, where the approximation is obtained by considering subsequences of partial sums of their Taylor expansion about a fixed centre. To be more precise we give the following definition:

Definition 1 Let $\Omega$ be a domain in the complex plane $\mathbb{C}$ and let $\zeta \in \Omega$. We say that a holomorphic function $f$ on $\Omega$ belongs to the collection $\mathcal{U}(\Omega, \zeta)$, of functions with universal Taylor series about $\zeta$, if, for each compact set $K \subset \mathbb{C} \backslash \Omega$ with connected complement and each continuous function $g: K \rightarrow \mathbb{C}$ which is holomorphic on $K^{\circ}$, there exists a subsequence of partial sums of the Taylor series of $f$ about $\zeta$ that converges uniformly to $g$ on $K$.

The above definition originated from a result of Nestoridis in 1996 in [33] who showed that, for most holomorphic functions on the unit disc (in the sense of Baire category), the partial sums of the Taylor expansion "overconverge" in such a chaotic manner outside the unit disc. This result was later extended by Melas and Nestoridis in [31], who established the following result:

Theorem 18 If $\Omega$ is simply connected, then $\mathcal{U}(\Omega, \zeta)$ is a dense $G_{\delta}$-set in the space of holomorphic functions on $\Omega$, endowed with the topology of the local uniform convergence. Moreover, the collection $\mathcal{U}(\Omega, \zeta)$ is independent of the choice of $\zeta$.

The situation becomes more complicated when $\Omega$ is multiply connected. There are examples of domains $\Omega$ for which the classes $\mathcal{U}(\Omega, \zeta)$, for each $\zeta \in \Omega$, are always empty, see [32], or always non-empty, see [30], while there are examples where the answer depends on the choice of the centre of expansion $\zeta$, see [G89]. Although there is a wide literature of partial results, the fundamental question of when universal Taylor series exist still remains open. A second main question that has attracted the interest of many researchers concerns the boundary behaviour of functions in $\mathcal{U}(\Omega, \zeta)$.

In order to solve problems related to the above questions, Stephen Gardiner introduced and developed novel techniques in this area which involved delicate potential theoretic arguments based on the Martin boundary, fine topology, harmonic measures and minimal thinness. His work yielded new insights about universal Taylor series and opened an alternative route for attacking problems where classical function theory techniques did not work. The initial point of connection of these two areas is that, for each holomorphic function $g$ on a domain, $\log |g|$ is subharmonic. This allows us to translate information about the convergence of the partial sums into the language of potential theory. This translation provides us with powerful machinery (estimates in terms of the Green functions, the Dirichlet problem, harmonic measure). Hence, whereas approximation theory techniques (like Mergelyan's theorem) help us to construct universal functions with "good" boundary behaviour, the role of potential theory is complementary: on one hand it allows us to determine geometrical and topological conditions for domains on which the existence of universal Taylor series is impossible and, on the other hand, it gives us certain results about "wild" boundary behaviour of such functions (for domains where they exist).

We highlight below some of his major contributions in the area: 


\section{Existence of universal Taylor series}

Stephen Gardiner developed in [G89], [G95] new techniques using various tools from potential theory such as harmonic measure and thinness to investigate sufficient conditions on $\Omega$ and $\zeta$, under which the existence of functions in $\mathcal{U}(\Omega, \zeta)$ is impossible. Two sample contributions in this direction are the following, where $D(\zeta, R)$ denotes the disc with centre $\zeta$ and radius $R$ and $\mathbb{D}=D(0,1)$.

Theorem 19 Let $\Omega$ be a domain in $\mathbb{C}$ and let $\zeta \in \Omega$. Then $\mathcal{U}(\Omega, \zeta)$ is empty if one of the following conditions is satisfied:

(i) $D(\zeta, R) \backslash \Omega$ is non-empty and polar for some $R>0$, and $\mathbb{C} \backslash \Omega$ is non-polar.

(ii) $\Omega \subset \mathbb{C} \backslash \overline{D(\xi, r)}$ and the set $D(\zeta, \rho) \backslash(\Omega \cup \overline{D(\xi, r)})$ is non-empty and polar for some $\rho$ satisfying $0<\rho \leq \sqrt{|\zeta-\xi|^{2}-r^{2}}$.

2. Properties of universal Taylor series

Stephen Gardiner showed that the class of universal Taylor series is not conformally invariant:

Theorem 20 Let $S=\{z:-1<\Re(z)<1\}$. Then there is a function $f \in \mathcal{U}(S, 0)$ such that, for any conformal mapping $\Phi: \mathbb{D} \rightarrow S$, the function $f \circ \Phi$ does not belong to $\mathcal{U}\left(\mathbb{D}, \Phi^{-1}(0)\right)$.

Moreover, he showed that for exterior Jordan domains with sufficiently smooth boundary the class $\mathcal{U}(\Omega, \zeta)$ depends on the choice of the centre of expansion $\zeta$ (unlike the simply connected case). More precisely, he established the following result:

Theorem 21 If $\Omega$ is the exterior domain of a Dini-smooth Jordan curve, then, for every $\zeta$, there exists $\zeta_{1} \in \Omega$ such that $\mathcal{U}(\Omega, \zeta) \backslash \mathcal{U}\left(\Omega, \zeta_{1}\right) \neq \emptyset$.

\section{Boundary behaviour of universal Taylor series}

In [G100], [G97], [G102] Stephen Gardiner combined potential theoretic tools from a wide spectrum to provide various results on the boundary behaviour of universal Taylor series. We include below three important instances of these results:

Theorem 22 (i) If $\Omega$ is the unit disc $\mathbb{D}$, then all functions in $\mathcal{U}(\Omega, 0)$ have a local Picard-type property.

(ii) If $\Omega$ is a simply connected domain, then all functions in $\mathcal{U}(\Omega, \zeta)$ are locally unbounded near every point of $\partial \Omega$.

(iii) For each domain $\Omega$, all functions in $\mathcal{U}(\Omega, \zeta$ ) are unbounded on $\Omega$ (whenever they exist).

4. New insights into the classical theory of power series

The strong machinery that Gardiner developed to solve problems about universal Taylor series brought new light into the classical theory of power series. In particular, in [G103] the authors obtain a new result on harmonic measures, which was used as a tool to "connect" the boundary behaviour of a Taylor series with the behaviour of its partial sums on the circle of convergence. More precisely, we have the following:

Theorem 23 For each holomorphic function $f$ on the unit disc, and for each subsequence $\left(S_{n_{k}}\right)$ of its Taylor polynomials about 0 , the nontangential limit of $f$ and $\lim _{k \rightarrow \infty} S_{n_{k}}$ coincide at almost all points of the unit circle where they simultaneously exist. 
In view of Plessner's theorem, the above result tells us that each Taylor series on the unit disc $\mathbb{D}$ which is universal on some subset $E$ of $\partial \mathbb{D}$ of positive arclength measure, must have wild angular boundary behaviour at almost every point of $E$. Finally, the authors proved analogues of these results for universal Dirichlet series in their subsequent paper [G106].

\section{Potential theory on cylindrical sets}

H. A. Schwarz introduced in 1869 (see [42]) the reflection principle for analytic functions, providing a very strong and useful tool in complex analysis and conformal mapping theory. Moreover he established reflection formulae along analytic curves based on the concept of the Schwarz function, see [23]. In all these cases a reflection law yields an analytic extension to a predefined larger domain and provides a simple formula for this extension. Since harmonic functions in two variables are locally real parts of analytic functions these methods lead to a reflection principle for harmonic functions which continuously vanish on a relatively open subset of a real-analytic boundary curve.

In higher dimensions the Schwarz reflection formula readily generalizes to give harmonic extension across a relatively open subset of a hyperplane or a sphere. For the hyperplane $\Gamma=\left\{\left(x_{1}, \ldots, x_{n}\right) \in \mathbb{R}^{n}: x_{1}=0\right\}$ the reflection is given by

$$
R_{\Gamma}\left(x_{1}, \ldots, x_{n}\right)=\left(-x_{1}, x_{2}, \ldots, x_{n}\right)
$$

Assume that $\Omega$ is a domain such that $R_{\Gamma}(x) \in \Omega$ for all $x \in \Omega$ and let $\Omega_{+}, \Omega_{0}, \Omega_{-}$ denote the set of all points $x \in \Omega$ where $x_{1}$ is respectively positive, zero, negative. Then for any harmonic function $h$ on $\Omega_{-}$such that $h(x) \rightarrow 0$ for $x \rightarrow y \in \Omega_{0}$ there exists a harmonic extension $\widetilde{h}$ defined on $\Omega$ by the formula

$$
\widetilde{h}(x)=-h\left(R_{\Gamma}(x)\right)
$$

A similar result is true for the sphere $\mathbb{S}^{n-1}=\left\{\left(x_{1}, \ldots, x_{n}\right) \in \mathbb{R}^{n}:\|x\|=1\right\}$ where the reflection is given by

$$
R_{\mathbb{S}^{n-1}}\left(x_{1}, \ldots, x_{n}\right)=\frac{x}{\|x\|^{2}} \text { for } x \neq 0 .
$$

Assume that $\Omega$ is a domain such that $R_{\mathbb{S}^{n-1}}(x) \in \Omega$ for all $x \in \Omega$ and let $\Omega_{+}, \Omega_{0}, \Omega_{-}$ denote the set of all points $x \in \Omega$ where $\|x\|$ is respectively smaller, equal, larger than 1. Then for any harmonic function $h$ on $\Omega_{-}$such that $h(x) \rightarrow 0$ for $x \rightarrow y \in \Omega_{0}$ there exists a harmonic extension $\widetilde{h}$ defined on $\Omega$ by

$$
\tilde{h}(x)=-\|x\|^{2-n} \cdot h\left(R_{\mathbb{S}^{n-1}}(x)\right) .
$$

In contrast to the two-dimensional case, no simple reflection results for other real-analytic hypersurfaces $\Gamma=\{x \in \Omega: f(x)=0\}$ have been found, where $f$ is 
assumed to be real-analytic on a domain $\Omega$ in $\mathbb{R}^{n}$ with non-vanishing gradient. In order to be more precise, we define

$$
\Omega_{-}=\{x \in \Omega: f(x)<0\} \quad \text { and } \quad \Omega_{+}=\{x \in \Omega: f(x)>0\}
$$

and we say that a point-to-point reflection exists for a pair $\left(x_{0}, x_{1}\right) \in \Omega_{-} \times \Omega_{+}$if there exists a constant $K=K\left(x_{0}, x_{1}\right)$ such that

$$
h\left(x_{1}\right)=K\left(x_{0}, x_{1}\right) h\left(x_{0}\right)
$$

for any harmonic function $h: \Omega_{-} \rightarrow \mathbb{R}$ which vanishes continuously on $\Gamma$. In [10] D. Khavinson and P. Ebenfelt proved that for odd dimension $n$ there exists point-to-point reflection for a real-analytic hypersurface $\Gamma$ only if $\Gamma$ is part of a hyperplane or sphere.

Beyond spheres and ellipsoids, cylinders form a class of examples which are important for applications and still accessible for explicit computations. Dima Khavinson raised the question whether a function which is harmonic on a circular cylinder in $\mathbb{R}^{3}$ and vanishes on the boundary must automatically have a harmonic extension to the whole of space. This issue has particular significance for the study of the singularities of solutions of the Dirichlet problem on cylindrical domains with entire boundary data $f$.

Dima's question was the starting point for a cooperation between Stephen and of one of the authors leading to a series of papers on the subject. In [G104] a positive answer to Dima's question is given:

Theorem 24 Let $n \geq 3$ and $B^{\prime}=\left\{x^{\prime} \in \mathbb{R}^{n-1}:\left\|x^{\prime}\right\|<1\right\}$ the unit ball of $\mathbb{R}^{n-1}$, and let $a>0$. If $h$ is harmonic on the finite cylinder $C_{a}:=B^{\prime} \times(-a, a)$ and continuously vanishes on the curved part of the boundary, i.e. on $\partial B^{\prime} \times(-a, a)$, then $h$ has $a$ harmonic extension $\widetilde{h}$ to $\mathbb{R}^{n-1} \times(-a, a)$.

The proof of this result is based on a detailed analysis of the Green function $G\left(x^{\prime}, x_{n}\right)$ of the infinite cylinder $C=C_{\infty}$ (so $a=\infty$ ) with pole $y=\left(y^{\prime}, y_{n}\right)$ which has been studied for dimension $n=3$ for a long time, see [9]. Writing $x=\left(x^{\prime}, x_{n}\right) \in \mathbb{R}^{n-1} \times \mathbb{R}$, the Green function for $n \geq 4$ can be described by a double series expansion of the form

$$
G(x)=\sum_{k=0}^{\infty} P_{k}^{\left(\frac{n-3}{2}\right)}\left(\frac{\left\langle x^{\prime}, y^{\prime}\right\rangle}{\left\|x^{\prime}\right\|\left\|y^{\prime}\right\|}\right) \sum_{m=1}^{\infty} D_{k, m}\left(\left\|x^{\prime}\right\|,\left\|y^{\prime}\right\|\right) e^{-j_{v_{k}, m}\left|x_{n}-y_{n}\right|}
$$

Here $P_{k}^{(\lambda)}(t)$ for $\lambda>0$ is the ultraspherical polynomial of degree $k$ and

$$
D_{k, m}\left(\left\|x^{\prime}\right\|,\left\|y^{\prime}\right\|\right)=v_{k} \frac{\left\|x^{\prime}\right\|^{\frac{3-n}{2}}\left\|y^{\prime}\right\|^{\frac{3-n}{2}} J_{v_{k}}\left(j_{v_{k}, m}\left\|y^{\prime}\right\|\right) J_{v_{k}}\left(j_{v_{k}, m}\left\|x^{\prime}\right\|\right)}{\rho_{v_{k}, m} \int_{0}^{1} J_{v_{k}}^{2}\left(j_{v_{k}, m} t\right) t d t},
$$


where $J_{v}(x)$ is the Bessel function of order $v$ of the first kind and $j_{v, m}$ is the $m$ th positive zero of $J_{v}$, and finally

$$
v_{k}=\frac{n-3}{2}+k
$$

For $n=3$ a similar formula to (9) holds, interpreting $P^{(\lambda)}$ as a limiting case for $\lambda \rightarrow 0$ leading to the Chebyshev polynomial $T_{k}(t)$.

The convergence of the series is rather delicate for $n=3$, and for $n \geq 4$ one needs to use mollifiers to provide a mathematical meaning of the infinite series in (9). Carslaw interprets (9) as the limiting case of the Green functions $G_{a}\left(x^{\prime}, x_{n}\right)$ of a finite cylinder $C_{a}$. For the Green function $G_{a}$ he provides a representation as a triple series - the sum of all normalized $L^{2}$-eigenfunctions of the Laplacian for the cylinder $C_{\alpha}$ divided by the eigenvalue. Most arguments in [9] are justified in a heuristic way. The paper [G104] provides a rigorous treatment.

A key observation is now that the Green function $G\left(x^{\prime}, x_{n}\right)$ for the pole $\left(y^{\prime}, y_{n}\right) \in C$ can be extended to a larger, naturally defined, domain. Clearly $G\left(x^{\prime}, x_{n}\right)$ has a singularity at $\left(y^{\prime}, y_{n}\right)$ and it can be shown $G\left(x^{\prime}, x_{n}\right)$ can be harmonically extended for all $\left(x^{\prime}, x_{n}\right)$ with $\left\|y^{\prime}\right\|<\left\|x^{\prime}\right\|<2-\left\|y^{\prime}\right\|$. This fact is crucial for the proof of the following result, which deals with harmonic functions defined on an open subset close to the curved boundary of a finite cylinder.

Theorem 25 Let $a>0$ and $\phi:[-a, a] \rightarrow[0,1)$ be continuous. If $h$ is harmonic on the set

$$
\left\{\left(x^{\prime}, x_{n}\right) \in \mathbb{R}^{n-1} \times \mathbb{R}:\left|x_{n}\right|<a \text { and } \phi\left(x_{n}\right)<\left\|x^{\prime}\right\|<1\right\}
$$

and continuously vanishes on $\partial B^{\prime} \times(-a, a)$, then $h$ extends to a harmonic function on the set

$$
\left\{\left(x^{\prime}, x_{n}\right):\left|x_{n}\right|<a \text { and } \phi\left(x_{n}\right)<\left\|x^{\prime}\right\|<2-\phi\left(x_{n}\right)\right\} \text {. }
$$

A striking aspect of this result is that, although we know that no point-to-point reflection principle applies to the function $h$, the domain of the harmonic extension is nevertheless formed by reflection along the radii of the cylinder.

Theorems 24 and 25 both concern outward harmonic extension through a cylindrical surface. A natural question is whether some kind of inward extension for the exterior cylinder is possible. One technical obstacle is the fact that a description of the Green function of the exterior cylinder seems to be unknown.

Now we discuss simultaneous inward and outward extension for harmonic functions which vanish on two coaxial cylindrical surfaces, see [G110]:

Theorem 26 Let $a>0, b>1$ and $A_{b}^{\prime}=\left\{x^{\prime} \in \mathbb{R}^{n-1}: 1<\left\|x^{\prime}\right\|<b\right\}$. Let $h$ be $a$ harmonic function on $A_{b}^{\prime} \times(-a, a)$ which continuously vanishes on $\partial A_{b}^{\prime} \times(-a, a)$. Then $h$ has a harmonic extension to the set

$$
\left\{\left(x^{\prime}, x_{n}\right):\left|x_{n}\right|<a,\left\|x^{\prime}\right\|>e^{\left(\left|x_{n}\right|-a\right) / b}\right\} \text {. }
$$

A simple limiting argument as $a \rightarrow \infty$ gives the following result. 
Theorem 27 Let $\Omega_{b}=\left\{\left(x^{\prime}, x_{n}\right): 1<\left\|x^{\prime}\right\|<b\right\}$ be the infinite annular cylinder for $b>1$. If $h$ is harmonic on $\Omega_{b}$ and $h$ continuously vanishes on $\partial \Omega_{b}$, then $h$ has $a$ harmonic extension to $\left(\mathbb{R}^{n-1} \backslash\left\{0^{\prime}\right\}\right) \times \mathbb{R}$.

The last two results depend on the properties of the Green function for the infinite annular cylinder. In this case the Green function has a double series expansion analogous to (9) but the function $D_{k, m}\left(\left\|x^{\prime}\right\|,\left\|y^{\prime}\right\|\right)$ now has to be replaced by

$$
\widetilde{D}_{k, m}\left(\left\|x^{\prime}\right\|,\left\|y^{\prime}\right\|\right)=v_{k} \frac{\left\|x^{\prime}\right\|^{\frac{3-n}{2}}\left\|y^{\prime}\right\|^{\frac{3-n}{2}} U_{v_{k}}\left(\rho_{v_{k}, m},\left\|x^{\prime}\right\|\right) U_{v_{k}}\left(\rho_{v_{k}, m},\left\|y^{\prime}\right\|\right)}{\rho_{v_{k}, m} \int_{1}^{b} U_{v_{k}}^{2}\left(\rho_{v_{k}, m}, t\right) t d t} .
$$

Here $U_{v}$ is the cross-product of scaled Bessel functions

$$
U_{v}(\rho, r)=J_{v}(\rho r) Y_{v}(\rho b)-J_{v}(\rho b) Y_{v}(\rho r)
$$

of the Bessel functions $J_{v}$ and $Y_{v}$ of the first and second kind respectively, and $\rho_{v, m}$ is the $m$ th positive zero of $\rho \longmapsto U_{v}(\rho, 1)$. The function $r \mapsto U_{v}\left(\rho_{v, m}, r\right)$ clearly vanishes when $r=\left\|x^{\prime}\right\|=b$ or $r=\left\|x^{\prime}\right\|=1$. A key step towards establishing convergence properties of the Green function of $\Omega_{b}$ is to provide strong uniform estimates for $\rho_{v, m}$ of the form

$$
\rho_{v, m+1}-\rho_{v, m}>\frac{\pi}{2 b-1} \quad(n \geq 1, m \geq 2) .
$$

In the survey [G108] the reader may find a list of open problems related to this new direction of research in potential theory.

\section{Analytic content}

Throughout this section, for any bounded function $g: S \rightarrow \mathbb{C}$ we denote by $\|g\|_{S}$ the quantity $\sup _{S}|g|$. The analytic content of a bounded domain $\omega$ in the complex plane $\mathbb{C}$ such that $\partial \omega$ is the disjoint union of finitely many simple analytic curves, is defined as

$$
\lambda(\omega)=\inf \left\{\|\bar{z}-\phi\|_{\bar{\omega}}: \phi \in A(\omega)\right\} .
$$

Here, $A(\omega)$ denotes the collection of continuous functions on $\bar{\omega}$ that are analytic on $\omega$. Alexander [3] and Khavinson [22] obtained the following "sandwich" isoperimetric inequalities

$$
\frac{2 A}{P} \leq \lambda(\omega) \leq \sqrt{\frac{A}{\pi}}
$$

where $A$ and $P$ denote the area and perimeter of $\omega$, respectively. For further details, the interested reader may consult Gamelin and Khavinson [16], and Bénéteau and 
Khavinson [5]. It was shown in [16] that equality with the upper bound occurs if and only if $\omega$ is a disc. Recently, Abanov et al. [1] have shown that equality with the lower bound occurs if and only if $\omega$ is a disc or an annulus.

Rewriting $\lambda(\omega)$ as $\inf \left\{\|z-\bar{\phi}\|_{\bar{\omega}}: \phi \in A(\omega)\right\}$, it can be seen that a natural generalization of this quantity to smoothly bounded domains $\Omega$ in Euclidean space $\mathbb{R}^{n}$ $(n \geq 3)$ is given by

$$
\lambda(\Omega)=\inf \left\{\|x-f\|_{\bar{\Omega}}: f \in A(\Omega)\right\},
$$

where $A(\Omega)$ now denotes the space of harmonic vector fields

$$
f=\left(f_{1}, \ldots, f_{n}\right) \in C(\bar{\Omega}) \cap C^{1}(\Omega)
$$

and $\|f\|_{S}=\sup _{S}\|f\|$, where $\|f\|=\sqrt{f_{1}^{2}+\cdots+f_{n}^{2}}$. Thus $f$ satisfies $\operatorname{div} f=0$ and curl $f=0$, which means that

$$
\sum_{i=1}^{n} \frac{\partial f_{i}}{\partial x_{i}}=0 \quad \text { and } \quad \frac{\partial f_{j}}{\partial x_{k}}-\frac{\partial f_{k}}{\partial x_{j}}=0 \text { for all } j, k \in\{1, \ldots, n\} \text { on } \Omega
$$

The gradient $\nabla h$ of any function $h \in C^{2}(\Omega) \cap C^{1}(\bar{\Omega})$ with $\Delta h=0$ on $\Omega$ is a harmonic vector field, and the converse assertion is also true when $\Omega$ is simply connected.

Let $r_{\Omega}>0$ be chosen so that a ball of radius $r_{\Omega}$ has the same volume as $\Omega$. Gustafsson and Khavinson [19] established that if $\Omega$ is a bounded domain in $\mathbb{R}^{n}$ with volume $V$ such that $\partial \Omega$ is the disjoint union of finitely many smooth components with total surface area $P$, then there exists a constant $c_{n}>1$ such that

$$
\frac{n V}{P} \leq \lambda(\Omega) \leq c_{n} r_{\Omega}
$$

It was conjectured in [19] that the optimal upper bound in (13) is $r_{\Omega}$. This conjecture was proved by Stephen Gardiner, Marius Ghergu and Tomas Sjödin [G109]. More precisely, we have:

Theorem 28 Let $\Omega$ be a bounded domain in $\mathbb{R}^{n}$ with volume $V$ such that $\partial \Omega$ is the disjoint union of finitely many smooth components with total surface area $P$. Then

$$
\frac{n V}{P} \leq \lambda(\Omega) \leq r_{\Omega}
$$

Further, $\lambda(\Omega)=r_{\Omega}$ if and only if $\Omega$ is a ball.

We now turn our attention to the Bergman $p$-analytic content.

The Bergman p-analytic content $(1 \leq p<\infty)$ of a bounded planar domain $\Omega$ was introduced by Guadarrama and Khavinson [18] as $\lambda_{A^{p}}(\Omega)=\inf _{f \in A^{p}(\Omega)}\|\bar{z}-f\|_{p}$, where $\|\cdot\|_{p}$ is the usual $L^{p}(\Omega)$-norm and $A^{p}(\Omega)$ is the Bergman space of $L^{p}(\Omega)$ integrable holomorphic functions $f$ on $\Omega$. In the case where $p=2$, Fleeman and 
Khavinson [11] showed that, for any simply connected domain $\Omega$ with piecewise smooth boundary, the following sandwich inequality holds

$$
\sqrt{\rho(\Omega)} \leq \lambda_{A^{2}}(\Omega) \leq \frac{m(\Omega)}{\sqrt{2 \pi}}
$$

Here, $\rho(\Omega)$ denotes the torsional rigidity of $\Omega$ and $m$ is Lebesgue measure. Subsequently, Fleeman and Lundberg [12] showed that the left hand inequality in (14) is actually an equality for any bounded simply connected domain, and this relationship has been further exploited by Fleeman and Simanek [13]. Bell, Ferguson and Lundberg [4] established related inequalities concerning torsional rigidity and the norm of the self-commutator of a Toeplitz operator. The limiting case of Bergman $p$-analytic content where $p=\infty$ is the notion of analytic content that we discussed above.

Rewriting $\lambda_{A^{p}}(\Omega)$ as $\inf _{\phi \in A^{p}(\Omega)}\|z-\bar{\phi}\|_{p}$, we see that a natural generalization to bounded domains $\Omega$ in Euclidean space $\mathbb{R}^{n}(n \geq 2)$ is given by

$$
\lambda_{A_{p}}(\Omega)=\inf \left\{\|x-f\|_{\mathcal{L}_{p}}: f \in A_{p}(\Omega)\right\}(1 \leq p<\infty)
$$

where $A_{p}(\Omega)$ denotes the space of all harmonic vector fields $f=\left(f_{1}, \ldots, f_{n}\right)$ in $\mathcal{L}_{p} \cap C^{1}(\Omega)$,

$$
\mathcal{L}_{p}=\mathcal{L}_{p}(\Omega)=\left(L^{p}(\Omega)\right)^{n}, \quad\|f\|_{\mathcal{L}_{p}}=\left(\int_{\Omega}\|f\|^{p} d m\right)^{1 / p}
$$

and $\|\cdot\|$ is the usual Euclidean norm on $\mathbb{R}^{n}$. Thus $f$ satisfies $\operatorname{div} f=0$ and curl $f=0$ in the sense of (12).

We will assume in the following that $\Omega$ is smoothly bounded. Denote by $q$ the dual exponent of $p$, whence $1 / p+1 / q=1$ (or $q=\infty$ if $p=1$ ), and note that the dual space $\mathcal{L}_{p}^{*}$ can be identified with $\mathcal{L}_{q}$. When $q<\infty$ we denote by $W_{0}^{1, q}(\Omega)$ the closure of $C_{c}^{\infty}(\Omega)$ in the Sobolev space $W^{1, q}(\Omega)$. Since any function in $W^{1, \infty}(\Omega)$ has a Lipschitz representative, it is natural to denote by $W_{0}^{1, \infty}(\Omega)$ the subset of $W^{1, \infty}(\Omega)$ comprising those functions which vanish on $\partial \Omega$. We define

$$
Q_{q}(\Omega)=\sup _{u \in W_{0}^{1, q}(\Omega) \backslash\{0\}} \frac{n}{\|\nabla u\|_{\mathcal{L}_{q}}} \int_{\Omega} u d m \quad(1<q \leq \infty) .
$$

When $q<\infty$, the quantity $\left(Q_{q}(\Omega)\right)^{q}$ is known as the St. Venant $q$-functional of $\Omega$.

The result below concerning the case $p=2$ was obtained in [G111].

Theorem 29 If $\Omega \subset \mathbb{R}^{n}$ is a smoothly bounded domain, then $\lambda_{A_{2}}(\Omega)=Q_{2}(\Omega)$. Further, $\lambda_{A_{2}}(\Omega)=\sqrt{\rho(\Omega)}$ if and only if $\mathbb{R}^{n} \backslash \Omega$ is connected.

The next result was established in [G111] and provides an optimal lower bound for $\lambda_{A_{p}}(\Omega)$ for all $p$. 
Theorem 30 If $\Omega \subset \mathbb{R}^{n}$ is a smoothly bounded domain and $p \in[1, \infty)$, then

$$
Q_{q}(\Omega) \leq \lambda_{A_{p}}(\Omega)
$$

Further, equality holds if and only if either

(a) $p=2$, or

(b) $\Omega$ is a ball or an annular region.

In the following we will discuss the optimal upper bound for $\lambda_{A_{p}}(\Omega)$. Let $B(r)$ denote the open ball in $\mathbb{R}^{n}$ of centre 0 and radius $r$, and let $B=B(1)$. By the generalized Faber-Krahn inequality we have $Q_{q}(\Omega) \leq Q_{q}\left(B\left(r_{\Omega}\right)\right)$. The result below obtained in [G111] is new in all dimensions.

Theorem 31 If $\Omega \subset \mathbb{R}^{n}$ is a smoothly bounded domain and $p \in[1,2]$, then

$$
\lambda_{A_{p}}(\Omega) \leq Q_{q}\left(B\left(r_{\Omega}\right)\right)=\left(\frac{n}{n+p} m(B)\right)^{1 / p} r^{1+n / p} .
$$

Further, equality holds if and only if $\Omega$ is a ball.

It is also conjectured in [G111] that (17) remains valid for all $p \in[1, \infty)$.

Theorems 30 and 31 together yield the following isoperimetric sandwich for Bergman $p$-analytic content.

Corollary 1 If $\Omega \subset \mathbb{R}^{n}$ is a smoothly bounded domain and $p \in[1,2]$, then

$$
Q_{q}(\Omega) \leq \lambda_{A_{p}}(\Omega) \leq Q_{q}\left(B\left(r_{\Omega}\right)\right)
$$

\section{Compliance with ethical standards}

Conflict of interest The authors declare that they have no conflict of interest.

Open Access This article is distributed under the terms of the Creative Commons Attribution 4.0 International License (http://creativecommons.org/licenses/by/4.0/), which permits unrestricted use, distribution, and reproduction in any medium, provided you give appropriate credit to the original author(s) and the source, provide a link to the Creative Commons license, and indicate if changes were made.

\section{References}

1. Abanov, A., Bénéteau, C., Khavinson, D., Teodorescu, R.: A free boundary problem associated with the isoperimetric inequality. J. Anal. Math. arXiv:1601.03885

2. Aharonov, D., Schiffer, M.M., Zalcman, L.: Potato kugel. Israel J. Math. 40, 331-339 (1981)

3. Alexander, H.: Projections of polynomial hulls. J. Funct. Anal. 13, 13-19 (1973)

4. Bell, S.R., Ferguson, T., Lundberg, E.: Self-commutators of Toeplitz operators and isoperimetric inequalities. Math. Proc. R. Ir. Acad. 114A, 115-133 (2014)

5. Bénéteau, C., Khavinson, D.: The isoperimetric inequality via approximation theory and free boundary problems. Comput. Methods Funct. Theory 6, 253-274 (2006) 
6. Birkhoff, G.D.: Démonstration d'un théorème élémentaire sur les fonctions entières. C. R. Acad. Sci. Paris 189, 473-475 (1929)

7. Brelot, M.: On topologies and boundaries in potential theory. Lecture Notes in Mathematics 175. Springer, Berlin (1971)

8. Caffarelli, L.A., Karp, L., Shahgholian, H.: Regularity of a free boundary with application to the Pompeiu problem. Ann. Math. 151, 269-292 (2000)

9. Carslaw, H.S.: Integral equations and the determination of Green's functions in the theory of potential. Proc. Edinb. Math. Soc. 31, 71-89 (1913)

10. Ebenfelt, P., Khavinson, D.: On point to point reflection of harmonic functions across real-analytic hypersurfaces in $\mathbb{R}^{n}$. J. Anal. Math. 68, 145-182 (1996)

11. Fleeman, M., Khavinson, D.: Approximating $\bar{z}$ in the Bergman space. Recent progress on operator theory and approximation in spaces of analytic functions. Contemp. Math. 679, 79-90 (2016)

12. Fleeman, M., Lundberg, E.: The Bergman analytic content of planar domains. Comput. Methods Funct. Theory 17, 369-379 (2017)

13. Fleeman, M., Simanek, B.: Torsional rigidity and Bergman analytic content of simply connected domains. arXiv:1704.01997v1 (2017)

14. Fuglede, B.: Finely harmonic functions. Lecture Notes in Mathematics 289. Springer, Berlin (1972)

15. Fuglede, B.: Fine potential theory. Potential theory-surveys and problems (Prague, 1987). Lecture Notes in Mathematics 1344, pp. 81-97. Springer, Berlin (1988)

16. Gamelin, T.W., Khavinson, D.: The isoperimetric inequality and rational approximation. Am. Math. Mon. 96, 18-30 (1989)

17. Grosse-Erdmann, K.G.: Universal families and hypercyclic operators. Bull. Am. Math. Soc. 36(3), 345-381 (1999)

18. Guadarrama, Z., Khavinson, D.: Approximating $\bar{z}$ in Hardy and Bergman norms. Banach spaces of analytic functions. Contemp. Math. 454, 43-61 (2008)

19. Gustafsson, B., Khavinson, D.: On approximation by harmonic vector fields. Houst. J. Math. 20, 75-92 (1994)

20. Gustafsson, B.: On quadrature domains and an inverse problem in potential theory. J. Anal. Math. 55, 172-216 (1990)

21. Gustafsson, B., Sakai, M., Shapiro, H.S.: On domains in which harmonic functions satisfy generalized mean value properties. Potential Anal. 7, 467-484 (1997)

22. Khavinson, D.: Annihilating measures of the algebra $R(X)$. J. Funct. Anal. 58, 175-193 (1984)

23. Khavinson, D., Lundberg, E.: Linear holomorphic partial differential equations and classical potential theory. Mathematical Surveys and Monographs 232. American Mathematical Society, Providence, RI (2018)

24. Khavinson, D., Lundberg, E., Render, H.: Dirichlet's problem with entire data posed on an ellipsoidal cylinder. Potential Anal. 46, 55-62 (2017)

25. Khavinson, D., Shapiro, H.S.: Remarks on the reflection principle for harmonic functions. J. Anal. Math. 54, 60-76 (1990)

26. King, J.R., Lacey, A.A., Vázquez, J.L.: Persistence of corners in free boundaries in HeleShaw flow. Eur. J. Appl. Math. 6, 455-490 (1995)

27. Kondraškov, A.V.: On the uniqueness of the reconstruction of certain regions from their exterior gravitational potentials (Russian). Ill-posed Mathematical Problems and Problems of Mathematical Geophysics, Novosibirsk, pp. 122-129 (1976)

28. Lukeš, J., Malý, J., Zajíček, L.: Fine topology methods in real analysis and potential theory. Lecture Notes in Mathematics 1189. Springer, Berlin (1986)

29. MacLane, G.R.: Sequences of derivatives and normal families. J. Anal. Math. 2, 72-87 (1952)

30. Melas, A.: Universal functions on nonsimply connected domains. Ann. Inst. Fourier (Grenoble) 51, 1539-1551 (2001)

31. Melas, A., Nestoridis, V.: Universality of Taylor series as a generic property of holomorphic functions. Adv. Math. 157, 138-176 (2001)

32. Müller, J., Vlachou, V., Yavrian, A.: Universal overconvergence and Ostrowski-gaps. Bull. Lond. Math. Soc. 38, 597-606 (2006)

33. Nestoridis, V.: Universal Taylor series. Ann. Inst. Fourier (Grenoble) 46, 1293-1306 (1996)

34. Novikoff, P.S.: Sur le problème inverse du potentiel. C. R. (Dokl.) Acad. Sci. URSS (N.S.) 18, 165-168 (1938)

35. Pal, J.: Zwei kleine Bemerkungen. Tohoku Math. J. 6, 42-43 (1914) 
36. Sakai, M.: A moment problem on Jordan domains. Proc. Am. Math. Soc. 70, 35-38 (1978)

37. Sakai, M.: Applications of variational inequalities to the existence theorem on quadrature domains. Trans. Am. Math. Soc. 276, 267-279 (1983)

38. Sakai, M.: Regularity of a boundary having a Schwarz function. Acta Math. 166, 263-297 (1991)

39. Sakai, M.: Regularity of boundaries of quadrature domains in two dimensions. SIAM J. Math. Anal. 24, 341-364 (1993)

40. Sakai, M.: Small modifications of quadrature domains, vol. 206. Memoirs of the American Mathematical Society (2010)

41. Shapiro, H.S.: The Schwarz function and its generalization to higher dimensions. University of Arkansas Lecture Notes in the Mathematical Sciences 9. Wiley, New York (1992)

42. Schwarz, H.A.: Über einige Abbildungsaufgaben. J. Reine Angew. Math. 70, 105-120 (1869)

43. Shahgholian, H.: Convexity and uniqueness in an inverse problem of potential theory. Proc. Am. Math. Soc. 116, 1097-1100 (1992)

44. Zalcman, L.: Some inverse problems of potential theory. Contemp. Math. 63, 337-350 (1987)

Publisher's Note Springer Nature remains neutral with regard to jurisdictional claims in published maps and institutional affiliations. 


\section{Scientific bibliography of Stephen Gardiner}

[G1] The Dirichlet and Neumann problems for harmonic functions in half-spaces. J. London Math. Soc. (2) 24 (1981) 502-512.

[G2] Harmonic majorization of subharmonic functions in unbounded domains. Ann. Acad. Sci. Fenn. Math. 8 (1983) 43-54.

[G3] Half-spherical means and boundary behaviour of subharmonic functions in half-spaces. Hiroshima Math. J. 13 (1983) 339-348.

[G4] Local and global majorization of subharmonic functions. J. Analyse Math. 42 (1983) 175-184.

[G5] Representation and growth of subharmonic functions in half-spaces. Proc. London Math. Soc. (3) 48 (1984) 300-318.

[G6] Some Phragmén-Lindelöf and harmonic majorization theorems for subharmonic functions. J. Math. Anal. Appl. 102 (1984) 156-174 (with D. H. Armitage).

[G7] A maximum principle and results on potentials. J. Math. Anal. Appl. 108 (1985) 507-514.

[G8] Convexity and subsolutions of partial differential equations. Bull. London Math. Soc. 18 (1986) 41-43 (with M. Klimek).

[G9] Convexity and subharmonic functions. Irish Math. Soc. Bull. 16 (1986) 4855.

[G10] Generalized means of subharmonic functions. Ann. Acad. Sci. Fenn. Math. 11 (1986) 3-27.

[G11] Subharmonic functions in strips. Ark. Mat. 24 (1986) 175-190.

[G12] Uniqueness theorems for subharmonic functions in unbounded domains. Proc. Amer. Math. Soc. 99 (1987) 437-444.

[G13] Integrals of subharmonic functions over affine sets. Bull. London Math. Soc. 19 (1987) 343-349.

[G14] The growth of the hyperplane mean of a subharmonic function. J. London Math. Soc. (2) 36 (1987) 501-512 (with D. H. Armitage).

[G15] Subharmonicity of mean values of subharmonic functions. Proc. Roy. Irish Acad. Sect. A 87 (1987) 169-175.

[G16] Functions harmonic outside a manifold. Bull. London Math. Soc. 20 (1988) 136-138.

[G17] Growth properties of $p$ th means of potentials in the unit ball. Proc. Amer. Math. Soc. 103 (1988) 861-869.

[G18] Integrals of subharmonic functions. Irish Math. Soc. Bull. 22 (1989) 33-41.

[G19] Convexity and subharmonicity. Elem. Math. 44 (1989) 145-150.

[G20] Minimal harmonic functions on Denjoy domains. Proc. Amer. Math. Soc. 107 (1989) 963-970.

[G21] Local growth properties of superharmonic functions. Arch. Math. 54 (1990) 52-60.

[G22] The Martin boundary of NTA strips. Bull. London Math. Soc. 22 (1990) 163-166.

[G23] Fine limits of superharmonic functions. Math. Proc. Cambridge Phil. Soc. 108 (1990) 381-394. 
[G24] Removable singularities for subharmonic functions. Pacific J. Math. 147 (1991) 71-80.

[G25] Subharmonicity, the distance function, and $a$-admissible sets. Proc. Amer. Math. Soc. 112 (1991) 979-981.

[G26] Maximum principles for subharmonic functions. Math. Scand. 68 (1991) 210-220.

[G27] A short proof of Burdzy's theorem on the angular derivative. Bull. London Math. Soc. 23 (1991) 575-579.

[G28] A fine limit property of functions superharmonic outside a manifold. Compositio Math. 83 (1992) 239-249.

[G29] Angular limits of holomorphic functions which satisfy an integrability condition. Monatsh. Math. 114 (1992) 97-106.

[G30] Boundary behaviour of holomorphic and harmonic functions. Irish Math. Soc. Bull. 29 (1992) 19-30.

[G31] Positive harmonic majorization of the real part of a holomorphic function. Proc. Amer. Math. Soc. 117 (1993) 767-770.

[G32] Separation of points by classes of harmonic functions. Math. Proc. Cambridge Phil. Soc. 113 (1993) 561-571 (with D. H. Armitage and I. Netuka).

[G33] The Dirichlet problem with non-compact boundary. Math. Z. 213 (1993) 163-170.

[G34] Sets of determination for harmonic functions. Trans. Amer. Math. Soc. 338 (1993) 233-243.

[G35] Conditions for separately subharmonic functions to be subharmonic. Potential Anal. 2 (1993) 255-261 (with D. H. Armitage).

[G36] Uniqueness and extension theorems for subharmonic functions. J. London Math. Soc. 48 (1993) 515-525.

[G37] Superharmonic extension and harmonic approximation. Ann. Inst. Fourier (Grenoble) 44 (1994) 65-91.

[G38] Uniform and tangential harmonic approximation. In: Classical and Modern Potential Theory, ed. K. GowriSankaran et al., NATO ASI Series, Vol. 430, Kluwer, Dordrecht, 1994, pp. 185-198.

[G39] Global approximation in harmonic spaces. Proc. Amer. Math. Soc. 122 (1994) 213-221 (with M. Goldstein and K. N. GowriSankaran).

[G40] Tangential approximation in harmonic spaces. Indiana Univ. Math. J. 43 (1994) 1003-1112 (with M. Goldstein and K. N. GowriSankaran).

[G41] Carleman approximation by harmonic functions. Amer. J. Math. 117 (1995) 245-255 (with M. Goldstein).

[G42] Tangential harmonic approximation on relatively closed sets. Illinois J. Math. 39 (1995) 143-157.

[G43] Superharmonic extension from the boundary of a domain. Bull. London Math. Soc. 27 (1995) 347-352.

[G44] Uniform harmonic approximation of bounded functions. Trans. Amer. Math. Soc. 348 (1996) 251-265.

[G45] Representation of continuous functions as sums of Green functions. Proc. Amer. Math. Soc. 124 (1996) 1149-1157. 
[G46] Harmonic approximation. In: Potential Theory - ICPT94, ed. J. Král et al., de Gruyter, Berlin, 1996, pp. 51-65.

[G47] Uniform harmonic approximation with continuous extension to the boundary. J. Analyse Math. 68 (1996) 95-106.

[G48] Characterization of best harmonic and superharmonic $L^{1}$-approximants. $J$. Reine Angew. Math. 478 (1996) 1-15 (with D. H. Armitage, W. Haussmann and L. Rogge).

[G49] The Lusin-Privalov theorem for subharmonic functions. Proc. Amer. Math. Soc. 124 (1996) 3721-3727.

[G50] Cluster sets of harmonic functions at the boundary of a half-space. Bull. London Math. Soc. 29 (1997) 191-194.

[G51] Decomposition of approximable harmonic functions. Math. Ann. 308 (1997) 175-185.

[G52] Harmonic approximation and boundary behaviour. In: Multivariate Approximation: recent trends and results, ed. W. Haussmann et al., Akad. Verlag, Berlin, 1997, pp. 45-62.

[G53] Best one-sided $L^{1}$-approximation by harmonic functions. Manuscripta Math. 96 (1998) 181-194 (with D. H. Armitage, W. Haussmann and L. Rogge).

[G54] Mergelyan pairs for harmonic functions. Proc. Amer. Math. Soc. 126 (1998) 2699-2703.

[G55] Asymptotic values and minimal fine limits of subharmonic functions of slow growth. J. London Math. Soc. 57 (1998) 668-676.

[G56] Limits of superharmonic functions along parallel lines. In: Complex Analysis and Differential Eqs., ed. C. Kiselman, Uppsala University, 1999, pp. 162-168.

[G57] Best harmonic and superharmonic $L^{1}$-approximants in strips. J. Approx. Theory 100 (1999) 266-283 (with D. H. Armitage).

[G58] Boundary growth theorems for superharmonic functions. Ark. Mat. 37 (1999) 255-273.

[G59] Best one-sided $L^{1}$-approximation by harmonic and subharmonic functions. In: Advances in Multivariate Approximation, ed. W. Haussmann et al., Wiley, Berlin, 1999, pp. 43-56 (with D. H. Armitage).

[G60] Limits along parallel lines and the classical fine topology. J. London Math. Soc. (2) 59 (1999) 881-894 (with M. Essén).

[G61] Evaluation of superharmonic functions using limits along lines. Bull. London Math. Soc. 32 (2000) 209-213 (with H. Aikawa).

[G62] Growth properties of superharmonic functions along rays. Proc. Amer. Math. Soc. 128 (2000) 1963-1970.

[G63] Incomplete lattice sets that control the behaviour of entire harmonic functions. Math. Proc. Cambridge Phil. Soc. 129 (2000) 549-563 (with D. H. Armitage, W. Haussmann and L. Rogge).

[G64] Convergence of rational interpolants with preassigned poles. Constr. Approx. 17 (2001) 139-146.

[G65] Nontangential limits, fine limits and the Dirichlet integral. Proc. Amer. Math. Soc. 129 (2001) 3379-3387. 
[G66] Harmonic approximation and its applications. In: Approximation, Complex Analysis and Potential Theory, ed. N. Arakelian and P.M. Gauthier, NATO ASI Series, Kluwer, Dordrecht, 2001, pp. 191-219.

[G67] Boundary sets where harmonic functions may become infinite. Math. Ann. 323 (2002) 41-54 (with W. Hansen).

[G68] Balayage properties related to rational interpolation. Constr. Approx. 18 (2002) 417-426 (with C. Pommerenke).

[G69] An equilibrium measure characterization of circles. Forum Math. 14 (2002) 953-954.

[G70] Farrell sets for harmonic functions. Proc. Amer. Math. Soc. 131 (2003) 773779 (with M. Hanley).

[G71] Carleman approximation for superharmonic functions. Bull. London Math. Soc. 35 (2003) 92-96.

[G72] Approximation on the boundary and sets of determination for harmonic functions, Illinois J. Math. 47 (2003) 1115-1136 (with J. Pau).

[G73] Smooth potentials with prescribed boundary behaviour. Publ. Mat. 48(2004) 241-249 (with A. Gustafsson).

[G74] Completeness of spaces of harmonic functions under restricted supremum norms. Comput. Methods Funct. Theory 4 (2004) 43-45.

[G75] Sets of harmonicity for finely harmonic functions. Potential Anal. 21 (2004) 1-6.

[G76] Radial limits of harmonic functions on the unit disc. Proc. Amer. Math. Soc. 133 (2005) 1387-1389.

[G77] Pointwise convergence and radial limits of harmonic functions. Israel J. Math. 145 (2005), 243-256 (with A. Gustafsson).

[G78] Radial limits of harmonic functions. In: Potential Theory in Matsue. Adv. Stud. Pure Math. 44, Math. Soc. Japan, Tokyo, 2006, pp. 43-51.

[G79] Potential theory of the farthest point distance function. J. Analyse Math. 101 (2007) 163-177 (with I. Netuka).

[G80] Quadrature domains for harmonic functions. Bull. London Math. Soc. 39 (2007) 586-590 (with T. Sjödin).

[G81] The Riesz decomposition for finely superharmonic functions. Adv. Math. 214 (2007) 417-436 (with W. Hansen).

[G82] The farthest point distance function. In: Complex and Harmonic Analysis (ed. A. Carbery et al.), DEStech, Lancaster, PA, 2007, pp. 35-43 (with I. Netuka).

[G83] Asymptotic maximum principles for subharmonic functions. Comput. Methods Funct. Theory 8 (2008) 167-172.

[G84] Convexity and the exterior inverse problem of potential theory. Proc. Amer. Math. Soc. 136 (2008) 1699-1703 (with T. Sjödin).

[G85] Lipschitz continuity of the Green function in Denjoy domains. Ark. Mat. 46 (2008) 271-283 (with T. Carroll).

[G86] Potential theory in Denjoy domains. In: Analysis and Math. Physics (ed. B. Gustafsson and A.Vasiliev), Birkhäuser, Basel, 2009, pp. 143-166 (with T. Sjödin). 
[G87] Partial balayage and the exterior inverse problem of potential theory. In: Potential Theory and Stochastics in Albac, Theta, Bucharest, 2009, pp. 111123 (with T. Sjödin).

[G88] Champagne subregions of the unit ball with unavoidable bubbles. Ann. Acad. Sci. Fenn. Math. 35 (2010) 321-329 (with M. Ghergu).

[G89] Universal Taylor series for non-simply connected domains. C. R. Math. Acad. Sci. Paris 348 (2010) 521-524 (with N. Tsirivas).

[G90] Finely continuously differentiable functions. Math. Z. 266 (2010) 851-861.

[G91] Sets of determination for the Nevanlinna class. Bull. London Math. Soc. 42 (2010) 1115-1120.

[G92] Potential theory in the exterior of a cylindrical set. Indiana Univ. Math. J. 59 (2010) 1777-1792.

[G93] Coincidence of harmonic and finely harmonic functions. Potential Anal. 34 (2011) 81-88.

[G94] Two-phase quadrature domains. J. Analyse Math. 116 (2012) 335-354 (with T. Sjödin).

[G95] Existence of universal Taylor series for non-simply connected domains. Constr. Approx., 35 (2012) 245-257.

[G96] Recent progress on fine differentiability and fine harmonicity. In: Complex Analysis and Potential Theory, CRM Proc. and Lecture Notes, vol. 55, Amer. Math. Soc., Providence, RI, 2012, pp. 283-291.

[G97] Boundary behaviour of functions which possess universal Taylor series. Bull. London Math. Soc. 45 (2013) 191-199.

[G98] Universal Taylor series, conformal mappings and boundary behaviour. Ann. Inst. Fourier (Grenoble) 64 (2014) 327-339.

[G99] Quadrature domains and their two-phase counterparts. In: Harmonic and Complex Analysis and its Applications, A. Vasiliev (ed.), Trends in Mathematics, Birkhäuser, Basel, 2014, pp. 261-285 (with T. Sjödin).

[G100] Boundary behaviour of universal Taylor series. C. R. Math. Acad. Sci. Paris 352 (2014) 99-103 (with D. Khavinson).

[G101] Stationary boundary points for a Laplacian growth problem in higher dimensions. Arch. Rational Mech. Anal. 213 (2014) 503-526 (with T. Sjödin).

[G102] Boundary behaviour of universal Taylor series on multiply connected domains. Constr. Approx. 40 (2014) 259-279 (with M. Manolaki).

[G103] A convergence theorem for harmonic measures with applications to Taylor series. Proc. Amer. Math. Soc. 144 (2016) 1109-1117 (with M. Manolaki).

[G104] Harmonic functions which vanish on a cylindrical surface. J. Math. Anal. Appl. 433 (2016) 1870-1882 (with H. Render).

[G105] A reflection result for harmonic functions which vanish on a cylindrical surface. J. Math. Anal. Appl. 443 (2016) 81-91 (with H. Render).

[G106] Boundary behaviour of Dirichlet series with applications to universal series. Bull. London Math. Soc. 48 (2016) 735-744 (with M. Manolaki).

[G107] Taylor series, universality and potential theory. In: New Trends in Approximation Theory, J. Mashreghi et al. (eds.) Fields Institute Communications, Springer, New York, 2018, pp. 247-264. 
[G108] Extension results for harmonic functions which vanish on cylindrical surfaces. Anal. Math. Phys. 8 (2018) 213-220 (with H. Render).

[G109] Analytic content and the isoperimetric inequality in higher dimensions. $J$. Funct. Anal. 275 (2018) 2284-2298 (with M. Ghergu and T. Sjödin).

[G110] Harmonic functions which vanish on coaxial cylinders. J. Analyse Math. (to appear) (with H. Render).

[G111] Bergman analytic content. Indiana Univ. Math. J. (to appear) (with M. Ghergu and T. Sjödin).

[G112] On a conjecture of Král concerning the subharmonic extension of continuously differentiable functions. Math. Bohem. (to appear) (with T. Sjödin).

[G113] A characterization of annular domains by quadrature identities. Bull. London Math. Soc. (to appear) (with T. Sjödin). 